4. Weston, V. C., Jones, A. C., Bradbury, N., Fawthrop, F. \& Doherty, M. Clinical features and outcome of septic arthritis in a single UK health district 1982. Ann. Rheum. Dis. 58, 214-219 (1999).

5. Goldenberg, D. L., Brandt, K. D., Cohen, A. S. \& Cathcart, E. S. Treatment of septic arthritis: comparison of needle aspiration and surgery as initial modes of joint drainage. Arthritis Rheum. 18, 83-90 (1975).

6. Gupta, M. N., Sturrock, R. D. \& Field, M. A prospective 2-year study of 75 patients with adult-onset septic arthritis. Rheumatology
(Oxford) 40, 24-30 (2001).

7. Sammer, D. M. \& Shin, A. Y. Comparison of arthroscopic and open treatment of septic arthritis of the wrist. J. Bone Joint Surg. Am. 91, 1387-1393 (2009).

8. El-Sayed, A. M. Treatment of early septic arthritis of the hip in children: comparison of results of open arthrotomy versus arthroscopic drainage. J. Child. Orthop. 3, 229-237 (2008).

9. Matthews, C. M., Weston, V. C., Jones, A., Field, M. \& Coakley, G. Bacterial septic arthritis in adults. Lancet (in press).

\title{
THERAPY
}

\section{Hydroxychloroquine in SLE: old drug, new perspectives}

Thomas Dörner

\section{Antimalarial agents have long been used in the treatment of autoimmune diseases, despite uncertainty regarding the exact mechanisms underlying their various effects. Growing evidence that these drugs offer protection from major infections could stimulate more research into these mechanisms, with possible implications for therapy.}

Chloroquine, together with its hydroxyl derivative hydroxychloroquine, was initially used as an antimalarial agent, but is also useful in the treatment of autoimmune diseases such as rheumatoid arthritis and systemic lupus erythematosus (SLE). Chloroquine and hydroxychloroquine are approved for the treatment of SLE and have been shown to reduce the frequency of disease flares (particularly of lupus nephritis), contribute to the maintenance of remission, prolong the onset of disease and reduce the risk of complications. ${ }^{1-5}$ Beyond their immunomodulatory effects in SLE, these agents have also been shown to protect against thrombotic events, ${ }^{2}$ improve glucose and lipid profiles, and prevent renal damage, ${ }^{6}$ apparently resulting in reduced cardiovascular risk, for which SLE is an independent risk factor. Ultimately, an increased rate of survival has been reported in SLE patients taking antimalarial agents. ${ }^{3,4}$

Antimalarial agents are inexpensive, especially compared with treatments used more recently in SLE patients, and also have a good overall tolerability profile, with the exception of a risk of retinal toxicity. To date, antimalarial agents have not been reliably linked to an increased incidence of infections as has been known to occur with use of other immunosuppressive drugs. In this context, and surprisingly, a recent nested case-control study aiming to identify factors predictive of infectious complications ${ }^{7}$ reported that SLE patients treated with antimalarial agents had a lower risk of major infections (odds ratio [OR] 0.06, 95\% CI 0.02-0.18), whereas prednisone treatment (OR 1.12, 95\% CI 1.04-1.19) and lung involvement (OR 4.41, 95\% CI 1.0618.36) increased this risk. Interestingly, the median dose of prednisone in patients with major infections was $7.5 \mathrm{mg}$ per day, and each $10 \mathrm{mg}$ per day dose increase led to an 11-fold increase in infection risk. Cyclophosphamide exposure and overt lupus nephritis were not associated with enhanced infection risk in this study, probably because an insufficient number of patients was included.

Although this study by Ruiz-Irastorza and colleagues ${ }^{7}$ was limited by the inclusion of only 83 cases of SLE and 166 controls, it did show that the risk of major infections is 16 times lower in patients treated with antimalarial agents than those who were not. This effect of antimalarials is consistent with observations from two previous studies in SLE. ${ }^{4,8}$ One retrospective study of patients with lupus nephritis identified a lower frequency of infections among patients previously treated with antimalarial agents. ${ }^{4}$ Another study in patients with $\mathrm{SLE}^{8}$ found that hydroxychloroquine treatment led to a lower risk of major infections, giving an OR of 0.05 (95\% CI 0.01-0.23); this is comparable to that of the study by Ruiz-Irastorza and colleagues ${ }^{7}$ which was partly corrected for disease activity.
Although prospective studies are needed to confirm these data, an assessment of the value of antimalarial agents, especially hydroxychloroquine, requires a re-evaluation of their underlying mechanisms of action. Beyond their benefits of clinical prevention of lupus flares, increased patient survival, prevention of severe organ damage, thrombosis and bone mass loss, and possible use in pregnant patients, indicators for reduced major infections might shed new light on antimalarial agents as a 'magic therapeutic bullet'. Most noteworthy, a similar reduction in infections with hydroxychloroquine use has been observed in patients with rheumatoid arthritis, which suggests that the reduction of infections might not be restricted to SLE. ${ }^{9}$

Usually, a key factor in considering immunosuppressive therapy for the treatment of patients with SLE is a substantially increased risk of infections. Immunosuppressive drugs, most notably steroids, as well as SLE activity, the presence of lupus nephritis or low serum complement levels have all been shown to increase the rate of infections. Although the intimate interrelationship between disease activity and choice of therapy intensity is critical in systemic autoimmune disease, one rule of clinical practice says that intensive therapy in patients with very active disease increases the risk of infections. How are antimalarial agents able to break this rule?

Even if the data by Ruiz-Istorza et al. ${ }^{7}$ can be confirmed in clinical prospective studies, we are still left with uncertainty about the unique underlying immunomodulatory and anti-infective effects of antimalarial agents. Originally used as antiparasitic agents, antimalarial drugs have also been shown to have antimicrobial effects, ${ }^{10}$ at least in vitro, against bacteria, such as Tropheryma whippelii, Staphylococcus aureus, Legionella pneumophila, Mycobacterium species, Salmonella typhi, Escherichia coli and Borrelia burgdorferi, fungi, ${ }^{10}$ and viruses including HIV, severe acute respiratory syndrome (SARS) coronavirus and others. ${ }^{7,10}$ This remarkable combination of immunomodulatory effects and effects against a variety of microbes is noteworthy and indicates hitherto unknown pathways of immune intervention and protection, respectively.

Two major concepts have been postulated to explain the mechanisms of action of antimalarial agents. First, alkalinization of phagolysosomes kills intracellular bacteria and fungi and, second, blockade of several steps of viral entry and protein 
glycosylation prevents the spread of viruses. ${ }^{10}$ The antibacterial and antifungal effects of antimalarial agents appear to be linked to interference with antigen presentation, which is mediated by $\mathrm{pH}$-dependent iron deprivation and by increasing the phagolysosomal $\mathrm{pH}$; this ultimately leads to reduced immune system activation and inhibits the growth of intracellular organisms. ${ }^{10}$ Moreover, the basic intracellular $\mathrm{pH}$ induced by antimalarial agents could have antiviral effects and result in the blockade of hydrolases and posttranslational modifications, including inhibition of glycosylation of newly synthesized proteins. Notably, glycosylation inhibition might lead to interactions of chloroquine with sugar-modifying enzymes or glycosyltransferases and could account for effects on a number of processes that are sialic-acid dependent, including intracellular as well as extracellular functions (for example, binding to surface receptors).

A third and rather appealing concept hypothesizes that interference with innate immune activation, that is, by blocking Toll-like receptor (TLR) 3, TLR7 and TLR9, could be responsible for a number of effects of antimalarial agents. Thus, antimalarial agents can interfere with intracellular recognition of nucleic-acid-binding by TLR7 and TLR9, which are harbored in intracellular compartments and block the activation of interferon in SLE. Since TLR recognition is also involved in protection against infection, a tempting idea is that antimalarial agents lead to prominent TLR inhibition by changing the intracellular $\mathrm{pH}$.

Despite reports of the valuable clinical effects of antimalarial agents in SLE, we still need additional confirmation from clinical studies as well as more-detailed basic studies to decipher their mechanisms of action. Since only about $40-50 \%$ of patients with SLE are treated with antimalarial agents, the available data on these drugs demand reconsideration of their pleiotropic effects in SLE patients where not contraindicated. Ultimately, a better understanding of the individual effects of antimalarial agents could pave the way for innovative drugs that combine immunomodulatory and anti-infective potency, or that even provide new etiopathogenic insights into SLE.

Department of Medicine, Rheumatology and Clinical Immunology, Charité

Universitätsmedizin Berlin and Deutsches

Rheumaforschungszentrum, Charitéplatz 01,

10098 Berlin, Germany.

thomas.doerner@charite.de

doi:10.1038/nrrheum.2009.235
Competing interests

The author declares no competing interests.

1. James, J. A. et al. Hydroxychloroquine sulfate treatment is associated with later onset of systemic lupus erythematosus. Lupus 16, 401-409 (2007).

2. Kaiser, R., Cleveland, C. \& Criswell, L. A. Risk and protective factors for thrombosis in systemic lupus erythematosus: results from a large, multi-ethnic cohort. Ann. Rheum. Dis. 68, 238-241 (2009).

3. Ruiz-Irastorza, G. et al. Effect of antimalarials on thrombosis and survival in patients with systemic lupus erythematosus. Lupus 15 , 577-583 (2006).

4. Siso, A. et al. Previous antimalarial therapy in patients diagnosed with lupus nephiritis: influence on outcomes and survival. Lupus 17, 281-288 (2008).

5. Tsakonas, E. et al. A long-term study of hydroxychloroquine withdrawal on exacerbations in systemic lupus erythematosus. The Canadian Hydroxychloroquine Study Group. Lupus 7, 80-85 (1998).
6. Pons-Estel, G. J. et al. Protective effect of hydroxychloroquine on renal damage in patients with lupus nephritis: LXV, data from a multiethnic US cohort. Arthritis Rheum. 61, 830-839 (2009).

7. Ruiz-Irastorza, G., Egurbide, M. V., Olivares, N., Martinez-Berriotxoa, A. \& Aguirre, C. Vitamin D deficiency in systemic lupus erythematosus: prevalence, predictors and clinical consequences. Rheumatology (Oxford) 47, 920-923 (2008).

8. Bultink, I. E. M. et al. Deficiency of functional mannose-binding lectin is not associated with infections in patients with systemic lupus erythematosus. Arthritis Res. Ther. 8, R183 (2006).

9. Smitten, A. L. et al. The risk of hospitalized infection in patients with rheumatoid arthritis. J. Rheumatol. 35, 387-393 (2008).

10. Rolain, J. M., Colson, P. \& Raoult, D. Recycling of chloroquine and its hydroxyl analogue to face bacterial, fungal and viral infections in the 21st century. Int. J. Antimicrob. Agents 30, 297-308 (2007).

\title{
PSYCHOLOGY
}

\section{Emotional disclosure in RA: does it work?}

\author{
Joan L. Duda and Jet J. C. S. Veldhuijzen van Zanten
}

\section{What is the psychological and clinical effectiveness of a home-based emotional disclosure intervention? Results from a randomized, controlled trial of such an intervention in patients with rheumatoid arthritis could have implications for the future of therapeutic research and clinical practice.}

The capacity to regulate emotions is predictive of perceived health in patients with rheumatoid arthritis (RA). ${ }^{1}$ Negative emotions, in particular stress, tend to exacerbate disease symptoms in this population. Emotional disclosure is an intervention centered on the promotion of emotion regulation and of confronting negative emotional experiences. ${ }^{2}$ Purposeful writing or talking about emotional topics is thought to lead to a more adaptive cognitive restructuring of the traumatic event or events in question. Via such disclosure, the individual is expected to formulate a more coherent and meaningful sense of the stressful circumstances. ${ }^{3}$

Particularly within laboratory studies, support has been garnered for the effects of emotional disclosure on reductions in stress and on improvements in positive psychological outcomes, in both patient and control populations. Beyond these psychological effects, emotional disclosure is assumed to lead to physiological benefits and, specifically, is thought to have a role in enhancing immune function.
With the aim of testing this type of intervention in a 'real-world setting', van Middendorp et al. ${ }^{4}$ adapted an emotional disclosure protocol for implementation in the homes of patients with RA. Oral disclosure was requested across four, short (15 minute) weekly sessions. The effects of the emotional disclosure intervention were compared with a time-management treatment, in which patients were asked to provide oral descriptions of recent and upcoming activities. Assessments were taken at baseline, and at 1 week and 3 months postintervention. The primary outcome measure was psychological well-being, which was operationalized with respect to cheerful versus depressed mood, assessed by use of the Impact of Rheumatic Diseases on General Health and Lifestyle questionnaire. No differential effect in the primary outcome measure emerged, and the two groups did not vary with respect to clinical outcomes. However, differences between the groups were observed over time with respect to indicators of physiological functioning, including urinary cortisol levels 


\section{CORRECTION}

THERAPY: Hydroxychloroquine in SLE: old drug, new perspectives

Dörner, T.

Nat. Rev. Rheumatol. 6, 10-11 (2010)

In the News and Views article by Thomas Dörner in the January 2010 issue of Nature Reviews

Rheumatology, the citation for reference number 7 was incorrect. The correct citation is: Ruiz-Irastorza, G.

et al. Predictors of major infections in systemic lupus erythematosus. Arthritis Res. Ther. 11, R109 (2009). 